ARTICLE

https://doi.org/10.1038/s41467-019-09942-z

\title{
Circularly polarized light detection using chiral hybrid perovskite
}

Chao Chen ${ }^{1,2}$, Liang Gao ${ }^{1,2}$, Wanru Gao ${ }^{1,2}$, Cong Ge ${ }^{1,2}$, Xinyuan Du¹,2, Zha Li ${ }^{1,2}$, Ying Yang ${ }^{1,2}$, Guangda Niu ${ }^{1,2} \&$ Jiang Tang (B) 1,2

Circularly polarized light (CPL) detection is required in various fields such as drug screening, security surveillance and quantum optics. Conventionally, CPL photodetector needs the installation of optical elements, imposing difficulties for integrated and flexible devices. The established CPL detectors without optical elements rely on chiral organic semiconductor and metal metamaterials, but they suffer from extremely low responsivity. Organic-inorganic hybrid materials combine CPL-sensitive absorption induced by chiral organics and efficient charge transport of inorganic frameworks, providing an option for direct CPL detection. Here we report the CPL detector using chiral organic-inorganic hybrid perovskites, and obtain a device with responsivity of $797 \mathrm{~mA} \mathrm{~W}^{-1}$, detectivity of $7.1 \times 10^{11}$ Jones, 3-dB frequency of $150 \mathrm{~Hz}$ and one-month stability, a competitive combined feature for circularly polarized light detection. Thanks to the solution processing, we further demonstrate flexible devices on polyethylene terephthalate substrate with comparable performance.

\footnotetext{
${ }^{1}$ Sargent joint research center, Wuhan National Laboratory for Optoelectronics, Huazhong University of Science and Technology, Wuhan 430074 Hubei, P. R. China. ${ }^{2}$ School of Optical and Electronic Information, Huazhong University of Science and Technology, Wuhan 430074 Hubei, P. R. China.

Correspondence and requests for materials should be addressed to J.T. (email: jtang@mail.hust.edu.cn)
} 
olarization, the same as intensity and wavelength, is a fundamental property of light. Circularly polarized light (CPL) is a light that the electric field has the constant magnitude but its direction continuously rotates in a plane with a steady rate. When interacting with a substance, the change of polarization state reflects the properties of this substance such as chemical composition, structure symmetry, surface roughness and so on ${ }^{1}$. Thus, a sensitive photodetector not only reveals the morphology of the target, but also provides valuable information regarding its chemical and physical nature. In fact, many invertebrates such as mantis shrimp, cuttlefish, bees and crickets have developed a CPLsensitive system in their retina ${ }^{2}$ to enhance visual contrast and differentiate substances for better navigation, communication and other applications. Polarization sensitive photodetector is thus highly valuable for application in many fields such as drug screening $^{3}$, security surveillance ${ }^{4}$, remote sensing ${ }^{5}$ and quantum optics $^{6}$. Because of the lack of intrinsic chirality in inorganic semiconductors such as $\mathrm{Si}$ and InGaAs, conventional CPLsensitive photodetector inevitably requires the installation of a quarter-wave plate and a linear polarizer on top of the non-chiral photodetectors. This strategy enables sensitive discrimination of polarized light despite at the price of increased cost and imposing some difficulties for integration and miniaturization.

One obvious solution is to find chiral sensitive materials that could naturally differentiate left-handed circularly polarized (LCP) and right-handed circularly polarized (RCP) light. We name the detection of CPL by the photodetector itself as direct CPL detection, while those require the assistance of optical elements as indirect CPL detection. Direct CPL detection has intrinsic advantages over indirect strategies for large-scale integration and for flexible electronics because of the removal of bulky wave plates (normally made of birefringent quartz, mica crystals and polymer sheet). Yet, to our best knowledge, there are only two papers in the literature reporting direct CPL detection to date $^{7,8}$. In 2013, A. Campbell et al. demonstrated a field-effect phototransistor employing chiral helicene for direct CPL detection with responsivity of $10 \mathrm{~mA} \mathrm{~W}^{-1}$ at a wavelength of $365 \mathrm{~nm}^{7}$. Two years later, J. Valentine et al. successfully built silver metamaterials with chiral structure and obtained distinctive optical absorption toward RCP and LCP light at a communication interesting wavelength of $1340 \mathrm{~nm}^{8}$. They further showed direct CPL detection based on hot electron injection but suffered from low responsivity of $2.2 \mathrm{~mA} \mathrm{~W}^{-1}$ mainly limited by the ultrafast decay of hot electrons in surface plasmons. Since responsibility reflects how sensitive a photodetector responds toward incident light and the commercial Si photodiode has a responsivity of approximately $1 \mathrm{~A} \mathrm{~W}^{-1}$ (ref. ${ }^{9}$ ), such a low responsivity of the available direct CPL detectors will certainly impede their practical applications, especially for weak CPL light detection and highdefinition imaging.

We pursued therefore the goal of CPL detection via seeking other suitable chiral materials. For a direct CPL photodetector to perform well, the absorber needs to combine handedness sensitive optical absorption and efficient charge transport simultaneously. Organic molecules are preferred for optical absorption because chiral organic compounds with strong circular dichroism (CD) are abundant in nature. For example, all amino acids show chirality except glycine ${ }^{10}$; the large biological molecules, such as proteins, DNA and RNA, and many natural products, such as chlorophyll copper sodium ${ }^{11}$ and carvone ${ }^{12}$, are known for their CPL-sensitive absorption. On the other hand, inorganic compounds generally enjoy high carrier mobility thanks to the transport within their bands. Combination of organic molecules with inorganic compounds could be an ideal strategy to overcome their own shortcomings and obtain a synergy for sensitive CPL detection. Decoration of inorganic quantum dots (QDs) with chiral organic ligands have been successfully engineered in the past decade to obtain CPL-sensitive optical absorption ${ }^{13}$, however, no such film has been applied for direct CPL detection possibly due to the insulating organic ligands that impede efficient charge transfer between QDs ${ }^{14}$. An alternative, probably more promising, is to construct organic-inorganic hybrid semiconductors. One prominent example is the recently emerged lead halide perovskites, which possess low-cost solution processing ${ }^{15}$, strong defect tolerance ${ }^{16}$, and long carrier diffusion length ${ }^{17}$, and have demonstrated outstanding performance for photovoltaics ${ }^{15}$, light emitting diodes ${ }^{18}$ and visible ${ }^{19}$, and $\mathrm{X}$-ray ${ }^{20,21}$ detector application. Very recently, by simply replacing the methylammonium with chiral amines or even other chiral organic molecules into A sites ${ }^{22-24}$, as-synthesized chiral halide perovskites not only possess handedness sensitive absorption by the chiral organics ${ }^{25}$, but also inherit the exceptional material and optoelectronic properties of the inorganic $\left(\mathrm{PbI}_{6}\right)^{4-}$ octahedral framework, a perfect combination for direct CPL detection.

We showcase here the synthesis of chiral organic-inorganic hybrid perovskites and their successful application for direct CPL photodetectors. We choose chiral $\alpha$-phenylethylamine ( $\alpha$-PEA) to synthesize chiral perovskites, because the $\pi$ bond in the benzene ring of $a$-PEA would facilitate columbic interaction between chiral amines and $\left(\mathrm{PbI}_{6}\right)^{4-}$ matrix, enhancing CPL-sensitive absorption, and also because right-hand ( $R$ - $\alpha$-PEA, Fig. 1a) and left-hand (S-a-PEA, Fig. 1b) enantiomers are commercially available with low $\operatorname{cost}^{26}$. One additional advantage is that $\alpha$ phenylethylamine hydroiodide [( $\alpha-P E A) I]$ has good solubility in dimethylformamide (DMF) and dimethylsulfoxide (DMSO) and hence permits easy single crystal growth and thin film fabrication.

\section{Result}

Single crystal fabrication. Single crystals of $\mathrm{ABX}_{3}$-type perovskite ( $R$ - and $S$ - $\alpha$-PEA) $\mathrm{PbI}_{3}$ were first produced by the inverse temperature crystallization method ${ }^{27}$. Three millimoles ( $\alpha$-PEA)I and $3 \mathrm{mmol} \mathrm{PbI}_{2}$ were dissolved in $45 \% \mathrm{HI}$ solution $(6 \mathrm{~mL})$ at $90^{\circ} \mathrm{C}$. Then the solution was slowly cooled from 90 to $40{ }^{\circ} \mathrm{C}$ with a cooling rate of $5^{\circ} \mathrm{C}$ per hour. After $10 \mathrm{~h}$, some yellow crystals formed from the solution (Supplementary Fig. 1), which were collected for further analysis. Single crystal diffraction experiments were performed; crystal structures were solved by Olex2 software ${ }^{28}$ (Supplementary Table 1, Fig. 1c, d) and are consistent with literature ${ }^{25}$. The crystals exhibit one-dimensional (1D) structure with face sharing $\left(\mathrm{PbI}_{6}\right)^{4-}$ octahedral chains surrounded by the chiral ( $\alpha$-PEA) ${ }^{+}$cations. The $(R$ - and $S$ - $\alpha$-PEA) $\mathrm{PbI}_{3}$ have a chiral space group of $\mathrm{P} 2{ }_{1} 2_{1} 2_{1}$ assigned to orthorhombic crystal system ${ }^{25}$, so they have only two-fold screw symmetry. Note that the chiral a-PEA stays closely to the vertex of $\left(\mathrm{PbI}_{6}\right)^{4-}$ octahedron enabling strong interaction between chiral organics and achiral inorganic parts. We failed to synthesize the racemic single crystal [denoted as ( $\left.r a c-\alpha-\mathrm{PEA} \mathrm{PbI}_{3}\right)$ ] because in our growing conditions only (rac- $\alpha-\mathrm{PEA})_{2} \mathrm{PbI}_{4}$ was produced. To confirm the structures, we also carried out powder X-ray diffraction (XRD) analysis on the ground powder of ( $R$ - and $S$-PEA) $\mathrm{PbI}_{3}$ single crystals, and all diffraction peaks were well consistent with the simulated results (Fig. 1e). An expanded XRD pattern is shown in Supplementary Fig. 2 for better peak resolution. The strongest diffraction peak at $8.27^{\circ}$ is assigned to (002) crystal face where $\left(\mathrm{PbI}_{6}\right)^{4-}$ octahedral chains stack in parallel.

Device design and film fabrication. For a photoconductive CPL photodetector, maximized value of $\Delta n / n$ (where $\Delta n$ and $n$ are equal to $n_{\mathrm{L}}-n_{\mathrm{R}}$ and $\left(n_{\mathrm{L}}+n_{\mathrm{R}}\right) / 2$, respectively; $n_{\mathrm{L}}$ and $n_{\mathrm{R}}$ are carrier concentration generated by LCP and RCP light illumination, respectively) should be pursued to enhance device 
a<smiles>CC(N)c1ccccc1</smiles>
$R$ - $\alpha$-PEA

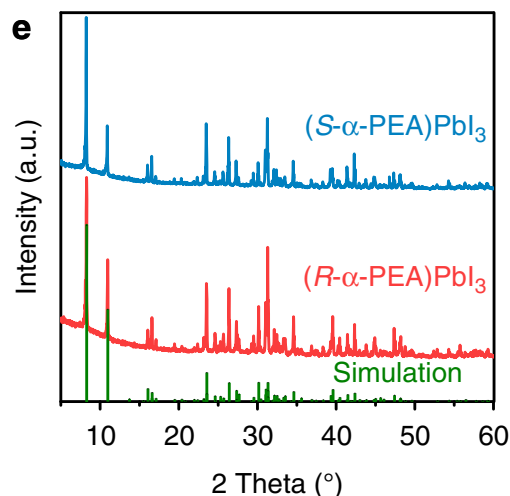

b<smiles>CC(N)c1ccccc1</smiles>

$S-\alpha-P E A$ c

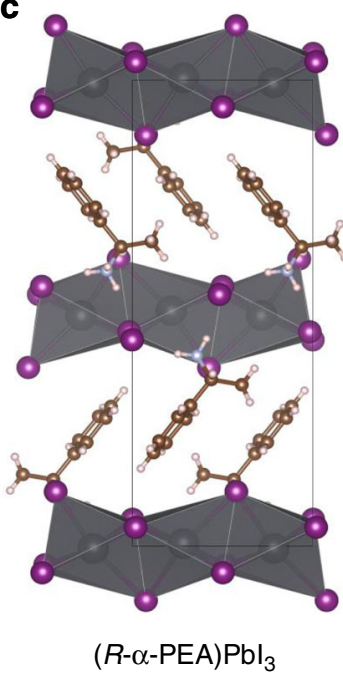

d

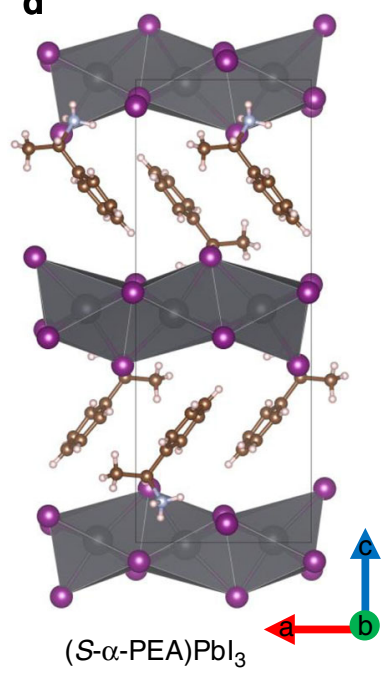

Fig. 1 The crystal structures and properties of our chiral perovskites. a, b Molecular structures of $R$ - and $S-\alpha-P E A$. $\mathbf{c}$, d The crystal structure of $(R$ - and $S$ - $\alpha$ $\mathrm{PEA}) \mathrm{Pbl}_{3}$. The gray, rose red, golden yellow, light blue, and fresh pink dots stand for $\mathrm{Pb}, \mathrm{I}, \mathrm{C}, \mathrm{N}$, and $\mathrm{H}$ atoms, respectively. e Powder XRD patterns of ( $R$ and $\mathrm{S}-\alpha-\mathrm{PEA}) \mathrm{Pbl}_{3}$. The simulated XRD peaks are shown as a comparison

sensitivity. $\Delta n / n$ as a function of ( $\alpha-\mathrm{PEA}) \mathrm{PbI}_{3}$ thickness $(d)$ can be addressed as (Supplementary Note 1):

$$
\frac{\Delta n}{n} \approx \frac{\Delta \alpha d}{\exp \left(\alpha_{\mathrm{L}} d\right)-1}
$$

where $\alpha_{\mathrm{L}}$ and $\alpha_{\mathrm{R}}$ are the absorption coefficient of LCP and RCP light, respectively; $\Delta \alpha$ is equal to $\alpha_{\mathrm{L}}-\alpha_{\mathrm{R}}$. When the active layer is too thick, the exponential term of $\exp \left(\alpha_{\mathrm{L}} d\right)$ in the denominator can dramatically reduce the value of $\Delta n / n$ (Supplementary Fig. 3). This means all incident LCP and RCP photons can be fully absorbed, resulting in no photocurrent difference between LCP and RCP illumination. Therefore, the thick single crystal (approximately $1 \mathrm{~mm}$ ) is unsuitable for CPL photodetector. Herein, films with appropriate thickness (equal to $1 / \alpha_{\mathrm{L}}$ as shown in Supplementary Fig. 3) are expected to obtain maximum $\Delta n / n$.

Luckily, the low-dimensional perovskites are prone to assemble themselves into highly-oriented and high-quality films by spincoating technique ${ }^{29}$. The precursor was prepared by dissolving ( $R$ - or $S$ - $\alpha$-PEA) $\mathrm{PbI}_{3}$ single crystal in DMF solution with the concentration of $0.3 \mathrm{~mol} \mathrm{~L}^{-1}$ to fabricate $\left(R\right.$ - or $S$ - $\alpha$-PEA) $\mathrm{PbI}_{3}$ films. The racemic precursor $\left(0.3 \mathrm{~mol} \mathrm{~L}^{-1}\right)$ prepared by mixing $(R$ - and $S$ - $\alpha-\mathrm{PEA}) \mathrm{PbI}_{3}$ precursor with a ratio of $1: 1$ was used for (rac-a-PEA) $\mathrm{PbI}_{3}$ films. XRD patterns of ( $R$-, $S$ - and rac- $\left.\alpha-\mathrm{PEA}\right)$ $\mathrm{PbI}_{3}$ films showed sharp diffraction peaks which can be assigned to (002), (004), (006), and (008) planes (Fig. 2a), indicating the highly preferred orientation along $c$-axis, the axis perpendicular to the plane of the film (Supplementary Fig. 4). The small full width at half maximum of these peaks also revealed high crystallinity. Two impurity peaks traceable to (002) and (004) of $(\alpha-\mathrm{PEA})_{2} \mathrm{PbI}_{4}$ phase ${ }^{22}$ appeared in the (rac-a-PEA) $\mathrm{PbI}_{3}$ films due to the competitive formation of this secondary phase. Atomic force microscope measurement suggested that (rac-a-PEA) $\mathrm{PbI}_{3}$ film was likely composed of mixed domains of $(R-\alpha-\mathrm{PEA}) \mathrm{PbI}_{3}$ and (S- $\alpha-P E A) \mathrm{PbI}_{3}$ (Supplementary Fig. 5). All the films showed strong excitonic absorption at $374 \mathrm{~nm}$ (Fig. 2b), a signature of the strong quantum confinement experienced by the $\left(\mathrm{PbI}_{6}\right)^{4-}$ chains in these materials with $1 \mathrm{D}$ crystal structure ${ }^{30}$. Both $(R-$ or $S$ - $\alpha$ $\mathrm{PEA}) \mathrm{PbI}_{3}$ films showed strong $\mathrm{CD}$ signals while ( $\left.\mathrm{rac}-\alpha-\mathrm{PEA}\right) \mathrm{PbI}_{3}$ film showed featureless absorption in the $\mathrm{CD}$ spectra. For the chiral perovskites, the $\mathrm{CD}$ spectra showed two intense peaks at 328 and $392 \mathrm{~nm}$ with the corresponding intensity of 183 and 210 mdeg, respectively. Ben-Moshe et al. discovered that the CD spectra can be calculated on the basis of the split excitonic transition states ${ }^{31,32}$, and each CD signal should have a corresponding excitonic transition ${ }^{22}$, implying two excitonic transitions in ( $\alpha-\mathrm{PEA}) \mathrm{PbI}_{3}$. The multiple excitonic transitions were also observed in other low-dimensional perovskites ( $\alpha$ $\mathrm{PEA})_{2} \mathrm{PbI}_{4}{ }^{22}$ and $\left(\mathrm{C}_{4} \mathrm{H}_{9} \mathrm{NH}_{3}\right)_{2} \mathrm{PbBr}_{4}{ }^{33}$. (rac- $\left.\alpha-\mathrm{PEA}\right) \mathrm{PbI}_{3}$ exhibited no $\mathrm{CD}$ signal because of the racemic mixture nature. The anisotropy factor of CD $\left(g_{\mathrm{CD}}\right.$, calculated as $\mathrm{CD} /(32980 \times$ absorbance) $)^{22}$ at $392 \mathrm{~nm}$ is $\sim 0.02$ (Fig. 2c), which is three times higher than that of $(\alpha-P E A)_{2} \mathrm{PbI}_{4}$ perovskite ${ }^{22}$ and one order of magnitude larger than that of ordinary chiral QDs ${ }^{34}$. Highresolution polarized optical microscopy study (Supplementary Fig. 6) confirms that $\mathrm{CD}$ is mainly originated from chiral perovskites, however, the weak dependence of $C D$ spectra on the rotation angle (Supplementary Fig. 7) suggests that linear birefringence or linear dichroism may also take effect to some extent.

The origin of chirality in hybrid perovskites. Now we discuss the origin of chirality in our hybrid perovskites. First, the CD signal positions of ( $R$ - or $S$ - $\alpha-P E A) P_{3}(328 \mathrm{~nm}$ and $392 \mathrm{~nm})$ are significantly different from the CD position of $\alpha$-PEA $(259 \mathrm{~nm}$, Supplementary Fig. 8). We thus propose that the chirality of the $\left(R\right.$ - and $S$ - $\alpha$-PEA) $\mathrm{PbI}_{3}$ perovskites comes from the chiral crystal structure $\left(\mathrm{P} 2{ }_{1} 2_{1} 2_{1} \text { space group }\right)^{35}$. From the view of quantum mechanics, the $\mathrm{CD}$ intensity is proportional to the rotational strength (R) as ${ }^{32,36}$ :

$$
\mathrm{CD} \propto \mathbf{R}=\operatorname{Im}\left[\left\langle\Psi_{\mathrm{s}}|\hat{\boldsymbol{\mu}}| \Psi_{\mathrm{j}}\right\rangle \cdot\left\langle\Psi_{\mathrm{j}}|\hat{\mathbf{m}}| \Psi_{\mathrm{s}}\right\rangle\right]=\operatorname{Im}\left[\boldsymbol{\mu}_{\mathrm{sj}} \cdot \mathbf{m}_{\mathrm{js}}\right]
$$

where $\Psi_{s}$ and $\Psi_{j}$ are the wave functions of the ground state and excited state; $\boldsymbol{\mu}_{\mathrm{sj}}$ and $\mathbf{m}_{\mathrm{js}}$ are the electric and magnetic transition dipole moments, respectively. To ensure a nonzero CD, the $\boldsymbol{\mu}_{\mathrm{s} j}$ and $\mathbf{m}_{\mathrm{js}}$ should be able to transform as the same irreducible representation (Supplementary Table 2) ${ }^{23}$. Only noncentrosymmetric chiral point groups satisfy the condition, therefore possessing $\boldsymbol{\mu}_{\mathrm{sj}} \neq 0$ and $\mathbf{m}_{\mathrm{js}} \neq 0$ and exhibiting $\mathrm{CD}^{23}$. For achiral point groups, because of their either inversion symmetry or central symmetry element, $\mathbf{R}$ is equal to zero, hence there is no optical activity. As mentioned above, ( $R$ - or $S$ - $\alpha-P E A) \mathrm{PbI}_{3}$ is assigned to non-centrosymmetric chiral space group of $\mathrm{P} 2{ }_{1} 2_{1} 2_{1}$, 

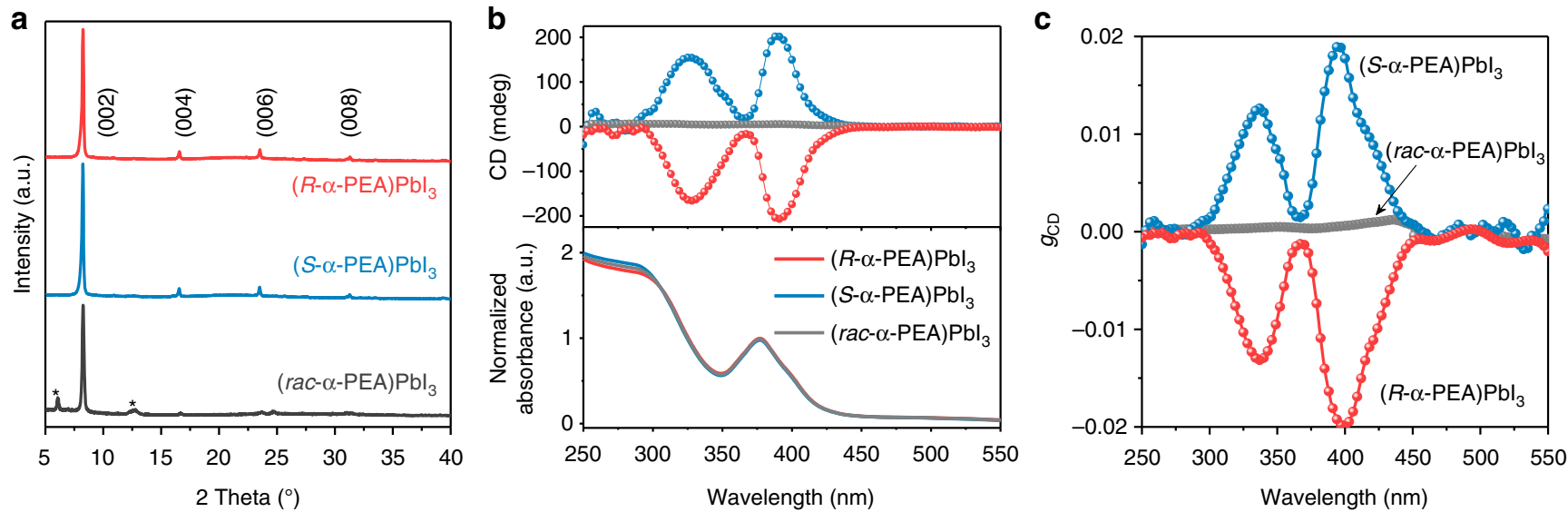

Fig. 2 The characterization of ( $\alpha-P E A)\left.P b\right|_{3}$ films. a XRD patterns of $\left.(R-, S-$, and rac- $\alpha-P E A) P b\right|_{3}$ films. The peaks marked with stars can be assigned to the $(002)$ and $(004)$ peaks of $(\alpha-P E A)_{2} \mathrm{Pbl}_{4}$ phase. $\mathbf{b} \mathrm{CD}$ and absorbance spectra of highly-oriented $\left.\left(R-, S_{-}\right.$, and rac- $\left.\alpha-\mathrm{PEA}\right) \mathrm{Pb}\right|_{3}$ films. c The $g_{\mathrm{CD}} \mathrm{Spectra}$ of $(R-$, $S-$, and $r a c-\alpha-P E A) \mathrm{Pbl}_{3}$ films

corresponding to point group of $\mathbf{D}_{2}$. Therefore the $(R$ - or $S$ - $\alpha$ $\mathrm{PEA}) \mathrm{PbI}_{3}$ exhibit chirality.

Device fabrication and performance. We assembled the highlyoriented ( $\alpha-\mathrm{PEA}) \mathrm{PbI}_{3}$ films into photodetector devices (Fig. 3a). $\mathrm{Au}$ electrodes defining a channel length of $10 \mu \mathrm{m}$ were prepared by thermal evaporation. Before measurement, we carefully calibrated our system to assure the same intensity of LCP and RCP illumination during all measurements (Supplementary Fig. 9). Figure $3 \mathrm{~b}$ showed the responsivity and photoconductor gain of $\left(R\right.$ - and $S$-a-PEA) $\mathrm{PbI}_{3}$ photodetectors under the CPL with the wavelengths of $365 \mathrm{~nm}(6.2 \mu \mathrm{W} \mathrm{cm}-2), 395 \mathrm{~nm}\left(5.0 \mu \mathrm{W} \mathrm{cm}{ }^{-2}\right)$, $430 \mathrm{~nm}\left(7.4 \mu \mathrm{W} \mathrm{cm}^{-2}\right)$, and $530 \mathrm{~nm}\left(79 \mu \mathrm{W} \mathrm{cm}^{-2}\right)$. The peak responsivity of our device can reach $0.12 \mathrm{~A} \mathrm{~W}^{-1}$ under $395 \mathrm{~nm}$ irradiation (corresponding to a photoconductor gain of $39.0 \%$ ). Our devices demonstrated notably different responsivity between LCP and RCP at the wavelengths of $395 \mathrm{~nm}$, however smaller difference at $365 \mathrm{~nm}$ and $430 \mathrm{~nm}$, and nearly no difference at $530 \mathrm{~nm}$, which are consistent with the CD and $g_{\mathrm{CD}}$ spectra. In analogy to the $g_{\mathrm{CD}}$, we defined the anisotropy factor of responsivity ( $g_{\text {res }}$, which describes the distinguishability to CPL) as:

$$
g_{\text {res }}=\frac{2\left(R_{\mathrm{L}}-R_{\mathrm{R}}\right)}{R_{\mathrm{L}}+R_{\mathrm{R}}}
$$

where $R_{\mathrm{L}}$ and $R_{\mathrm{R}}$ are the responsivity under LCP and RCP illumination, respectively. As long as the conversion and collection efficiency of absorbed photons to electron-hole pairs is the same, $g_{\text {res }}$ is in principle equal to $g_{\mathrm{CD}}$ (Supplementary Note 1 ). In our case, the maximum $g_{\text {res }}$ is 0.1 at the wavelength of $395 \mathrm{~nm}$, higher than $g_{\mathrm{CD}}$ of 0.02 at $395 \mathrm{~nm}$. This is possibly caused by the spindependent carrier transport and collection ${ }^{37}$. Patrick Odenthal et al. has reported that the $\mathrm{CPL}$ can generate spin-electron in $\mathrm{CH}_{3} \mathrm{NH}_{3} \mathrm{PbI}_{3}$ perovskite due to the spin-dependent optical selection rules ${ }^{38}$. In addition, caused by the large Rashba splitting in perovskite and ruled by the angular momentum conservation $^{23,39}$, the spin-up and spin-down electrons (holes) stay at different conduction band minimum (valence band maximum $)^{23,38}$. This may affect the spin-dependent carrier transport via carrier effective mass. The anisotropy factor of responsivity in our device is inferior to the chiral photodetector employing plasmonic metamaterials, which enjoys an extremely large $g_{\mathrm{CD}}$ of 0.9 and $g_{\text {res }}$ of 1.09 (the 1.09 value is calculated by ourselves based on the reported $R_{\mathrm{L}} / R_{\mathrm{R}}$ of 3.4$)^{7,8}$. Obviously, the $g_{\mathrm{CD}}$ of our device should be further improved in the future.
We also tested the linear dynamic region (LDR) of our device under unpolarized $395 \mathrm{~nm}$ light with light intensity ranging from 0.05 to $252 \mu \mathrm{W} \mathrm{cm} \mathrm{cm}^{-2}$. We did not choose $395 \mathrm{~nm}$ CPL light for LDR measurement because the maximum intensity of our light source is limited to $5.0 \mu \mathrm{W} \mathrm{cm}{ }^{-2}$ due to optical attenuation by the waveplate. The device exhibited wide LDR within nearly three orders of magnitude (Fig. 3d). The light intensity dependent responsivity and photoconductor gain were illustrated in Fig. 3d. The responsivity can reach $797 \mathrm{~mA} \mathrm{~W}^{-1}$ (corresponding to a photoconductor gain of $253 \%$ ), which is nearly two order of magnitude larger than the chiral molecule based ${ }^{7}$ and metamaterial based ${ }^{8} \mathrm{CPL}$ photodetectors. The frequency dependent noise spectrum in dark is showed in Fig. 3e, which showed strong $1 / f$ characteristics because its polycrystalline nature causes substantial carrier scattering at the grain boundaries ${ }^{40}$. Combining the measured noise current $\left(i_{\mathrm{n}}\right)$ at $1 \mathrm{~Hz}\left(2.5 \times 10^{-29} \mathrm{~A}^{2} \mathrm{~Hz}^{-1}\right)$ and the corresponding responsivity $\left(797 \mathrm{~mA} \mathrm{~W}^{-1}\right)$, we can calculate the detectivity $\left(D^{*}\right)$ through $D^{*}=R \sqrt{A} / i_{n}$ (where $R$ is responsivity, $A$ is the effective area of the detector $)^{41}$ as $7.1 \times 10^{11}$ Jones. The detectivity is impressive as commercial $\mathrm{Si}$ photodiode has a polarization-insensitive detectivity of approximately $10^{12}$ Jones $^{41}$. The $3-\mathrm{dB}$ frequency, defined as the frequency at which response dropped to half of the initial value, was approximately $150 \mathrm{~Hz}$ for $395 \mathrm{~nm}$ response, faster than the 30 frame per second required for imaging application. All combined, our direct CPL photodetector shows high sensitivity, low noise and fast response, a balanced and competitive performance for polarization sensitive imaging, manifesting the advantage of using chiral perovskites for direct $\mathrm{CPL}$ detection. Compared to the performance of $\mathrm{CH}_{3} \mathrm{NH}_{3} \mathrm{PbX}_{3}$ based CPL-insensitive photodetectors (e.g. best responsibility of approximately $10 \mathrm{AW}^{-1}, D^{*}$ of $10^{12}-10^{14}$ Jones $)^{19,42}$, there is a large room for further optimization of our CPL-sensitive photodetectors, which could be achieved by identifying better chiral perovskites, improving film quality and exploring appropriate device architecture.

The direct CPL based on chiral materials holds promise for flexible optoelectronic devices. Enabled by the low-temperature solution process, here we fabricate our direct CPL photodetector onto flexible polyethylene terephthalate (PET) substrate. Optical measurement of our perovskite film revealed two CD peaks at 330 and $385 \mathrm{~nm}$ (Fig. 4a), consistent with previous results on quartz. The noisy CD spectra from 300 to $320 \mathrm{~nm}$ were caused by the parasitic absorption of PET substrate. Encouragingly, the device also demonstrated CPL detection capability (Fig. 4b) with comparable $g_{\text {res }}$ of 0.08 ( 0.1 for the device on quartz substrate). 
a
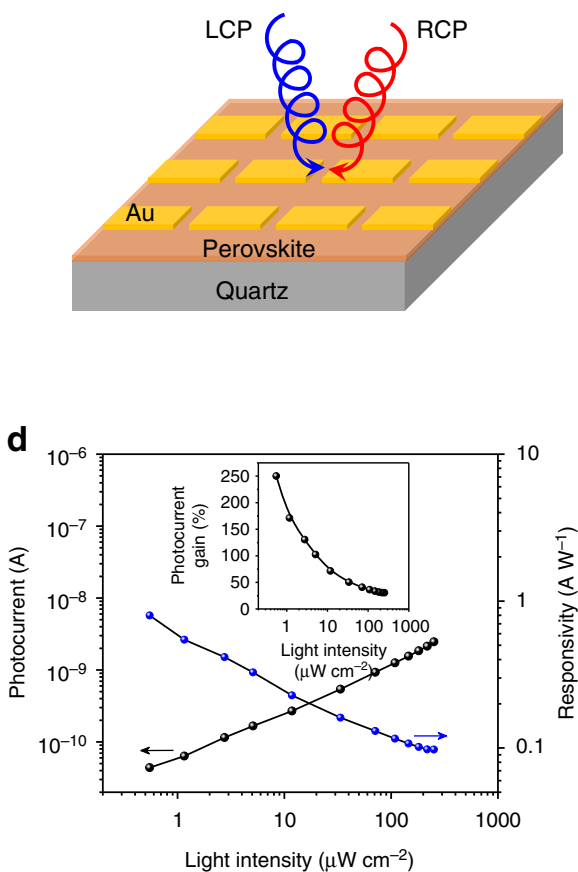

b

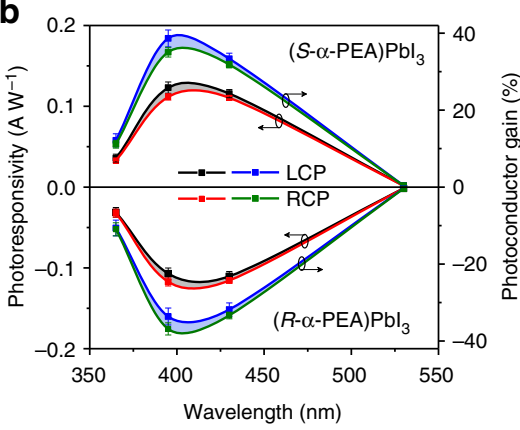

e

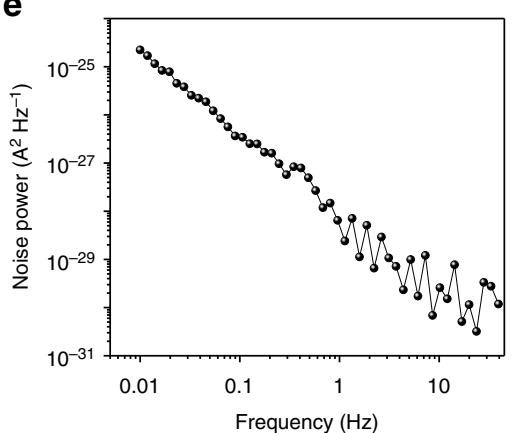

C

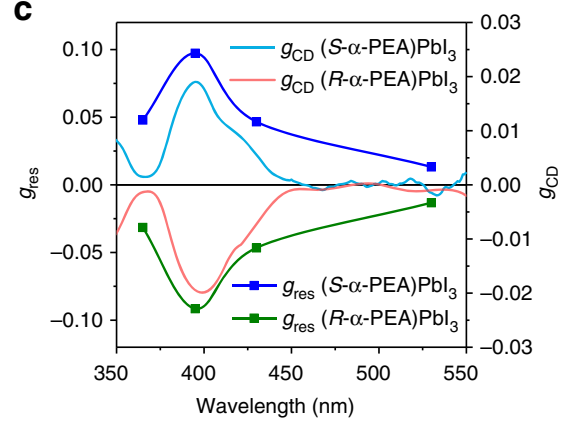

f

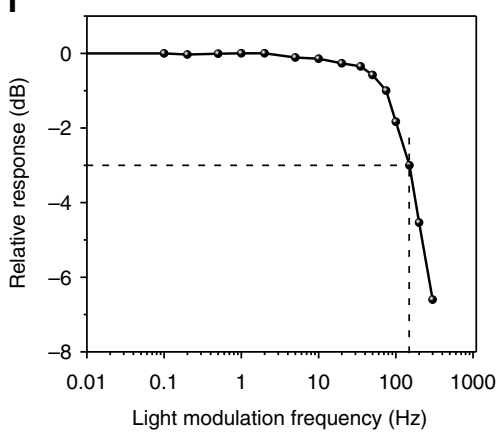

Fig. 3 The device performance of $(R-$ and $S-\alpha-P E A) P_{3} l_{3}$ film photodetector. a Schematic diagram of our photodetector. $\mathbf{b}$ The responsivity and photoconductor gain of ( $R$ - and S- $\alpha$-PEA)Pbl 3 device under LCP and RCP light at the wavelengths of 365, 395, 430, and 530 nm. The gray and cambridge blue area denotes the difference of responsivities and photoconductor gain under LCP and RCP irradiation, respectively, with identical intensity. $\mathbf{c}$ The wavelength dependent $g_{\text {res }}$ spectra. The $g_{\mathrm{CD}}$ spectra are also shown here in order to facilitate comparison. $\mathbf{d}$ Light intensity dependent LDR and responsivity at $20 \mathrm{~V}$ under $395 \mathrm{~nm}$ irradiance. The inset is the light intensity dependent photoconductor gain. e Measured current noise at various frequencies. $\mathbf{f}$ Normalized response of the photodetector versus the input signal frequency. The $-3-d B$ point is specified with the dash lines
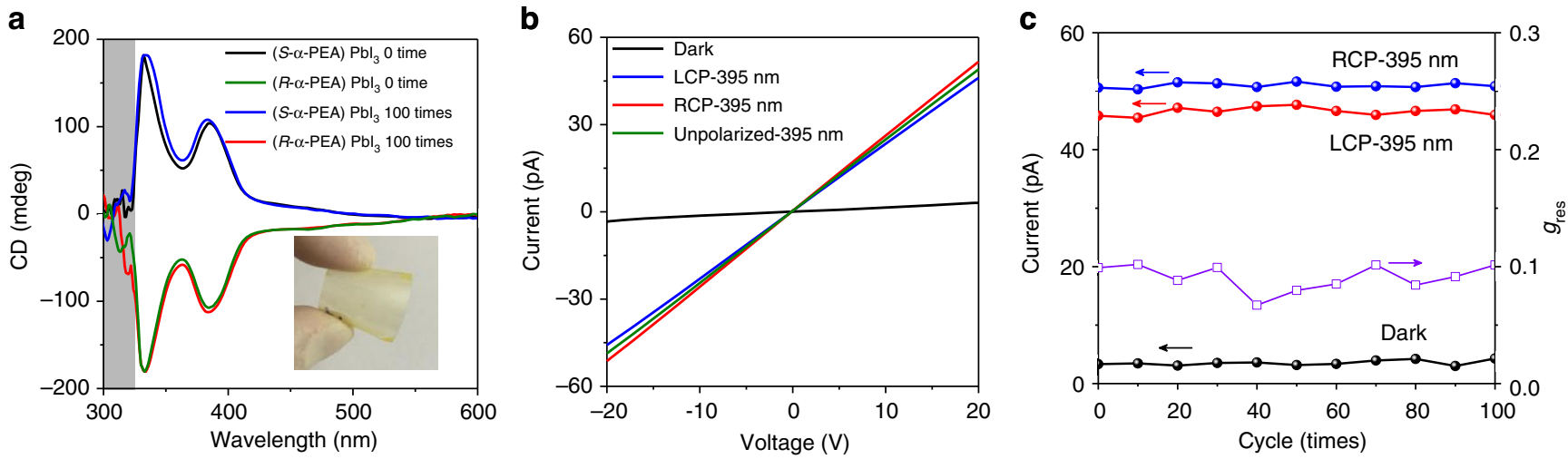

Fig. 4 Device performance of ( $\alpha$-PEA)Pbl $\left.\right|_{3}$ films on flexible PET substrate. a The CD spectra of ( $R$ - and $\left.S-\alpha-P E A\right)$ Pbl $\left.\right|_{3}$ films on PET substrates without bending and with 100-times bending. Inset is the digital photograph of the film on PET substrate. $\mathbf{b}$ The current-voltage curves of $(R-\alpha-P E A) P b l_{3}$ device under dark, LCP-395, RCP-395, and unpolarized-395 nm light illumination. The light intensity was $5 \mu \mathrm{W} \mathrm{cm}{ }^{-2}$. c The current under dark, LCP-395 and RCP$395 \mathrm{~nm}$ irradiation as well as $g_{\text {res }}$ as a function of folding cycles

The responsivity under LCP-395 $\mathrm{nm}, \mathrm{RCP}-395 \mathrm{~nm}$ and unpolarized-395 nm light illumination $\left(5.0 \mu \mathrm{W} \mathrm{cm} \mathrm{cm}^{-2}\right)$ are $0.090,0.098$, and $0.094 \mathrm{~A} \mathrm{~W}^{-1}\left(0.12 \mathrm{~A} \mathrm{~W}^{-1}\right.$ for the device on quartz substrate under $5.0 \mu \mathrm{W} \mathrm{cm}-2$ ). The current under dark, LCP-395 nm and RCP-395 nm light illumination as well as the $g_{\text {res }}$ as a function of folding times are shown in Fig. 4c. Both the CD spectra, dark current and photocurrent, and the anisotropy factor of responsivity $\left(g_{\text {res }}\right)$ exhibited negligible degradation upon 100 times of bending, confirming the robustness of our chiral perovskite films. We envision our work would enable many unexplored applications for flexible and/or wearable electronics.

We further monitored the XRD pattern, CD spectra and electrical stability of our $(S-\alpha-\mathrm{PEA}) \mathrm{PbI}_{3}$ film after stored in ambient without any encapsulation for 1 month to check its stability (Fig. 5). All of these characterizations revealed negligible degradation after the ambient storage. The device also exhibited no degradation when continuously operated for $2 \mathrm{~h}$ (Supplementary Fig. 10). Our chiral perovskites demonstrated similar stability compared to low-dimensional achiral perovskites ${ }^{22,30}$. Recent calculations revealed that the energy required to remove a phenethylammonium iodide from lowdimensional perovskite is $0.36 \mathrm{eV}$ higher than that of methylammonium iodide from 3D perovskite, reducing the desorption rate of organic molecules by 6 orders of magnitude ${ }^{30}$. This explains the environmental stability of our chiral hybrid perovskites. 
a

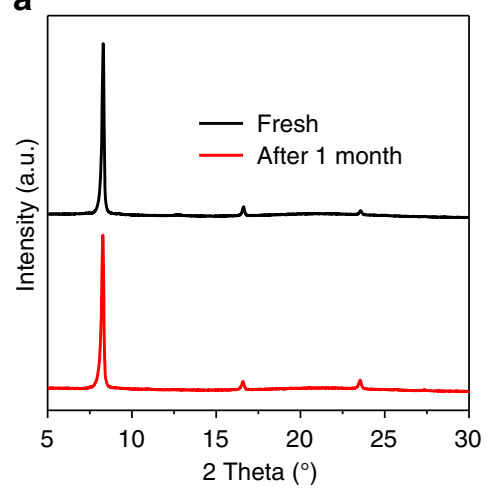

b

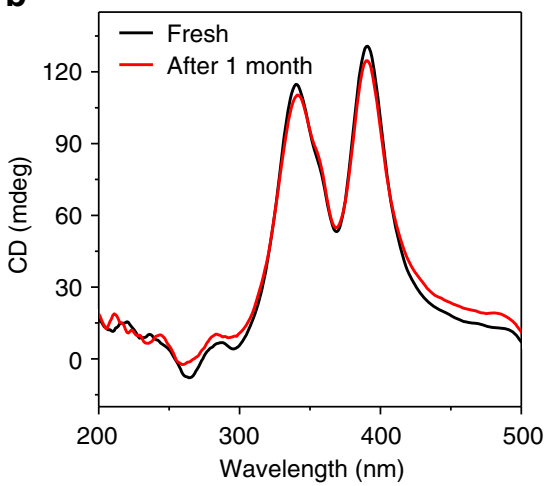

C

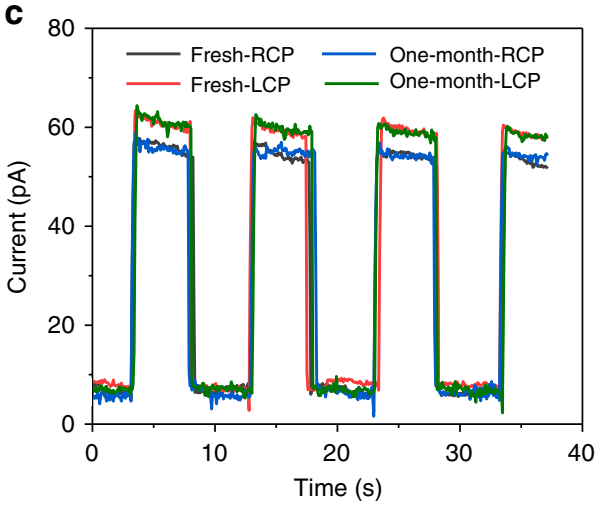

Fig. 5 The ambient stability of ( $R-\alpha-P E A)\left.P b\right|_{3}$ films. a XRD patterns, b CD spectra, and $\mathbf{c}$ Photoresponse of fresh and 1-month aged (S- $\alpha$-PEA)Pbl ${ }_{3}$ films under LCP and RCP $395 \mathrm{~nm}$ light. ( $\mathrm{S}-\alpha-\mathrm{PEA}) \mathrm{Pbl}_{3}$ films are stored in a laboratory environment without any encapsulation

\section{Discussion}

The moderate $g_{\text {res }}$ of our CPL detector need improvement for practical application. According to Eqs. (1) and (3), improvement in $g_{\mathrm{CD}}$ represents a straightforward strategy to boost $g_{\text {res }}$. We thus propose the following potential approaches to strengthen the chiral sensitivity of our hybrid perovskites CPL detector: (i) Seek organic amines with multiple chiral sites. Chirality transfer accounts for the chirality of perovskites ${ }^{23}$; amine with multiple chiral sites (such as threonine, isoleucine, 1,2-diphenylethylenediamine, etc.) could be explored into halide perovskites. (ii) Engineer a chiral octahedron and inorganic framework without mirror symmetry or centro-symmetry. Small B-site cation (such as $\mathrm{Cu}^{2+}, \mathrm{Ge}^{2+}, \mathrm{Sb}^{3+}$, etc.) and mixed anions at X-site $\left(\mathrm{Cl}^{-}, \mathrm{Br}^{-}, \mathrm{I}^{-}\right.$, $\mathrm{SCN}^{-}$, etc.) can significantly distort the $\left(\mathrm{BX}_{6}\right)^{4-}$ octahedron and potentially produce strong chirality and improve $g_{\mathrm{CD}}$ in principle. (iii) Produce mesoscopically chiral ordering. Similar to metamaterials ${ }^{8}$, structured halide perovskite films with characteristic lengths comparable to the wavelength of light enjoy strong chiroptical effect, leading to the improvement of $g_{\text {res }}$.

In summary, we successfully fabricated a direct CPL detector using chiral organic-inorganic hybrid ( $\alpha-\mathrm{PEA}) \mathrm{PbI}_{3}$ perovskites with an impressive responsivity of $795 \mathrm{~mA} \mathrm{~W}^{-1}$. This device showed a measured detectivity of $7.1 \times 10^{11}$ Jones, $3-\mathrm{dB}$ frequency of $150 \mathrm{~Hz}$ and high stability, a balanced and competitive device performance for potential polarization sensitive imaging applications. We also demonstrated the flexible CPL detector on PET substrate with high performance and robust flexibility. Moreover, chiral organic-inorganic hybrids combine the polarization sensitive absorption of chiral organics and efficient charge transfer of inorganics, providing a rich family of candidate materials for direct CPL detection. Such hybrids include halide perovskites incorporating other chiral amines such as benzedrine, (2-naphthyl)ethanammonium and sec-butylamine into A sites, and the large family of metalorganic frameworks (MOFs) materials containing chiral organics at the framework. We envision that direct CPL detection without optical elements would open an exciting realm for the easy realization of miniaturized polarization sensitive imaging systems.

\section{Methods}

Materials. $R$ - $\alpha$-phenylethylamine ( $R$ - $\alpha$-PEA, $99.0 \%$ gas chromatography purity, $98 \%$ enantiomeric excess), $S$ - $\alpha$ - phenylethylamine ( $S$ - $\alpha$-PEA, $99.0 \%$ gas chromatography purity), N,N-anhydrous dimethylformamide (DMF, 99.8\%), lead iodide $\left(\mathrm{PbI}_{2}, 99.9 \%\right)$ were purchased from Aladdin (Shanghai, China). Forty-five percent aqueous hydriodic acid (HI) solution $(99.95 \%)$ was purchased from Sinopharm Chemical Reagent Co., Ltd. (China).

Synthesis of ( $\boldsymbol{R}$ - and $\boldsymbol{S}$ - $\boldsymbol{\alpha}$-PEA)I. ( $R$ - $\alpha$-PEA)I (or (S- $\alpha$-PEA)I) was prepared by slowly mixing $R$ - $\alpha$-PEA (or $S$ - $\alpha$-PEA) $(5.1 \mathrm{~mL})$ with $\mathrm{HI}(5.8 \mathrm{~mL})$ in a $1: 1$ molar ratio under continuous stirring at $0^{\circ} \mathrm{C}$ for $2 \mathrm{~h}$. (R- $\alpha$-PEA)I (or $\left.(S-\alpha-\mathrm{PEA}) \mathrm{I}\right)$ was then crystallized by removing the solvent from a rotary evaporator at $80^{\circ} \mathrm{C}$ for $4 \mathrm{~h}$, washed three times in diethyl ether and collected by filtering. The white crystal was dried in vacuum for $24 \mathrm{~h}$ and then kept in a dark and dry environment for further use.

Growth of ( $\boldsymbol{R}$ - and $\boldsymbol{S}$ - $\alpha$-PEA)Pbl 3 single crystals. $0.75 \mathrm{~g}(3 \mathrm{mmol})$ of $(R-\alpha-P E A) \mathrm{I}$ (or $(S-\alpha-P E A) I)$ and $0.69 \mathrm{~g}(3 \mathrm{mmol})$ of $\mathrm{PbI}_{2}$ were fully dissolved in $6 \mathrm{ml}$ of a $45 \%$ HI solution at $90^{\circ} \mathrm{C}$. Then $1 \mathrm{~mL}$ simethicone was added to protect the precursor from air. The solution was slowly cooled from 90 to $40{ }^{\circ} \mathrm{C}$ with a cooling rate of $5{ }^{\circ} \mathrm{C}$ per hour. Many yellow crystals produced after $10 \mathrm{~h}$. These crystals were collected and washed by hexane to remove simethicone from the surface.

Fabrication of ( $\alpha$-PEA)PbI3 film. Quartz glass and polyethylene terephthalate (PET) were used as the substrate for rigid and flexible devices. Both substrates with the desired dimensions were cleaned in an ultrasonic cleaner using detergent, acetone, 2-propanol, ethanol, and deionized water in sequence for $30 \mathrm{~min}$ of each. Next, the substrate surface was cleaned by an ultraviolet-ozone cleaner for $10 \mathrm{~min}$. The precursor solutions for the chiral perovskites were prepared by dissolving the ( $R$ - or $S$ - $\alpha$-PEA) $\mathrm{PbI}_{3}$ single-crystal powders in DMF with the desired concentration $\left(0.3 \mathrm{~mol} \mathrm{~L}^{-1}\right)$. To form the films, $80 \mu \mathrm{L}$ of the precursor solution was spread on the cleaned surface of the substrate, and then spun at $1000 \mathrm{rpm}$ for $10 \mathrm{~s}, 3000 \mathrm{rpm}$ for $70 \mathrm{~s}$. Finally, the as-fabricated film was annealed at $95^{\circ} \mathrm{C}$ for $10 \mathrm{~min}$ on a hot-plate to induce crystallization.

Device preparation. Planar structured photoconductive detectors were fabricated by thermally evaporating $\mathrm{Au}$ electrodes onto ( $R$ - or $S$ - $\alpha-\mathrm{PEA}) \mathrm{PbI}_{3}$ films, and the channel between neighboring electrodes had a width of $0.5 \mathrm{~mm}$ and a length of $10 \mu \mathrm{m}$. The thermal evaporation was conducted using the electron beam and resistance evaporation thin film deposition system (Beijing Technol Science Co. Ltd.) under a vacuum pressure $5 \times 10^{-3} \mathrm{~Pa}$.

Material characterization. Powder XRD measurements were performed by grinding $\left(R\right.$ - and $S$ - $\alpha$-PEA) $\mathrm{PbI}_{3}$ into fine powders in a mortar using a Philips diffractometer (X pert pro MRD) with a step of $0.017^{\circ}$ and step time of $15.05 \mathrm{~s}$ from $5^{\circ}$ to $40^{\circ}$. The lines are $\mathrm{Cu} \mathrm{K}_{\alpha 1}$ and $\mathrm{Cu} \mathrm{K} \mathrm{K}_{22}$ with wavelengths of $1.540598 \AA$ and $1.544426 \AA$, respectively. The CD and extinction spectra were collected using a CD spectrometer (J-815, JASCO, Easton, MD, USA) at room temperature. The background was air, and the spectra were obtained at a scan rate of $200 \mathrm{~nm} \mathrm{~min}^{-1}$, with the data pitch being $1 \mathrm{~nm}$. For single crystal diffraction measurement, diffraction data were collected on a Bruker SMART 1K CCD with standard PILATUS3 R 200K detector. The X-ray source was generated by $\mathrm{Cu}$ MicroMax-003 microfocus sealed tube. The collection method is $\omega$ scans of $0.5^{\circ}$ width. Crystal structure was first analyzed by direct method with SHELXS solution program and then refined by SHELXL using least-squares method. All of nonhydrogen atoms' positions were located using difference Fourier method. Crystal data are listed in Supplementary Table 1. Powder diffraction XRD pattern of ( $\alpha$-PEA) $\mathrm{PbI}_{3}$ was simulated by software VESTA. For $\mu \tau$ test, the dark and light $(395 \mathrm{~nm}$, $37 \mu \mathrm{W} \mathrm{cm}{ }^{-2}$ ) current at $1 \mathrm{~V}$ to $100 \mathrm{~V}$ were measured by Agilent B1500A.

Detector performance measurement. The current-voltage and current-time curves were measured using Agilent B1500A in an electromagnetically shielded probe station. The light was generated from LEDs with wavelength of $365 \mathrm{~nm}$ (Thorlabs M365L2), $395 \mathrm{~nm}$ (Thorlabs M395L2), $430 \mathrm{~nm}$ (Thorlabs M430L3) and $530 \mathrm{~nm}$ (Thorlabs M450L3). Circularly polarized light was obtained by a linear polarizer (Thorlabs, LPVIS200-100-A) and quarter-wave plate (Thorlabs, AQWP05M-600). The intensity of LCP and RCP light was calibrated by standard Si detector (Newport 818-UV/DB) before each measurement with extreme caution (Supplementary Fig. 9). The 3-dB and noise power were measured by a preamplifier (SR570) and a lock-in amplifier (SR850, Stanford Research Systems). 


\section{Data availability}

The data that support the findings of this study are available from the corresponding author on request.

Received: 27 July 2018 Accepted: 25 February 2019

Published online: 26 April 2019

\section{References}

1. Longhurst, R. S. Geometrical and physical optics. (Orient BlackSwan Pvt. Ltd., Telangana, 1970).

2. Chiou, T.-H. et al. Circular polarization vision in a stomatopod crustacean. Curr. Biol. 18, 429 (2008).

3. H Brooks, W., C Guida, W. \& G Daniel, K. The significance of chirality in drug design and development. Curr. Top. Med. Chem. 11, 760 (2011).

4. Lin, S.-S., Yemelyanov, K. M., Pugh, E. N. \& Engheta, N. Polarization enhanced visual surveillance techniques. In Proceedings of IEEE International Conference on Networking, Sensing and Control 216-221 (Taipei, Taiwan, 2004)

5. Talmage, D. \& Curran, P. Remote sensing using partially polarized light. Int. J. Remote Sens. 7, 47 (1986).

6. Sherson, J. F. et al. Quantum teleportation between light and matter. Nature 443, 557 (2006).

7. Yang, Y., Da Costa, R. C., Fuchter, M. J. \& Campbell, A. J. Circularly polarized light detection by a chiral organic semiconductor transistor. Nat. Photonics 7, 634 (2013).

8. Li, W. et al. Circularly polarized light detection with hot electrons in chiral plasmonic metamaterials. Nat. Commun. 6, 8379 (2015).

9. Carey, J. E., Crouch, C. H., Shen, M. \& Mazur, E. Visible and near-infrared responsivity of femtosecond-laser microstructured silicon photodiodes. Opt. Lett. 30, 1773 (2005).

10. Bhushan, R. \& Brückner, H. Marfey's reagent for chiral amino acid analysis: a review. Amino acids 27, 231 (2004).

11. Houssier, C. \& Sauer, K. Circular dichroism and magnetic circular dichroism of the chlorophyll and protochlorophyll pigments. J. Am. Chem. Soc. 92, 779 (1970).

12. Lambert, J., Compton, R. \& Crawford, T. D. The optical activity of carvone: a theoretical and experimental investigation. J. Chem. Phys. 136, 114512 (2012).

13. Ma, W. et al. Chiral inorganic nanostructures. Chem. Rev. 117, 8041 (2017).

14. Tang, J. et al. Colloidal-quantum-dot photovoltaics using atomic-ligand passivation. Nat. Mater. 10, 765 (2011).

15. Yang, W. S. et al. High-performance photovoltaic perovskite layers fabricated through intramolecular exchange. Science 348, 1234 (2015).

16. Meggiolaro, D. et al. Iodine chemistry determines the defect tolerance of leadhalide perovskites. Energy Environ. Sci. 11, 702 (2018).

17. Dong, Q. et al. Electron-hole diffusion lengths $>175 \mu \mathrm{m}$ in solution-grown CH3NH3PbI3 single crystals. Science 347, 967 (2015).

18. Xiao, Z. et al. Efficient perovskite light-emitting diodes featuring nanometresized crystallites. Nat. Photonics 11, 108 (2017)

19. Dou, L. et al. Solution-processed hybrid perovskite photodetectors with high detectivity. Nat. Commun. 5, 5404 (2014).

20. Wei, H. et al. Sensitive X-ray detectors made of methylammonium lead tribromide perovskite single crystals. Nat. Photonics 10, 333 (2016)

21. Yakunin, S. et al. Detection of X-ray photons by solution-processed lead halide perovskites. Nat. Photonics 9, 444 (2015)

22. Ahn, J. et al. A new class of chiral semiconductors: chiral-organic-moleculeincorporating organic-inorganic hybrid perovskites. Mater. Horiz. 4, 851 (2017).

23. Long, G. et al. Spin control in reduced-dimensional chiral perovskites. Nat. Photonics 12, 528 (2018)

24. Georgieva, Z. N., Bloom, B. P., Ghosh, S. \& Waldeck, D. H. Imprinting Chirality onto the Electronic States of Colloidal Perovskite Nanoplatelets. Adv. Mater. 30, 1800097 (2018).

25. Billing, D. G. \& Lemmerer, A. Synthesis and crystal structures of inorganicorganic hybrids incorporating an aromatic amine with a chiral functional group. CrystEngComm 8, 686 (2006).

26. Hulst, R., de Vries, N. K. \& Feringa, B. L. a-Phenylethylamine based chiral phospholidines; new agents for the determination of the enantiomeric excess of chiral alcohols, amines and thiols by means of 31P NMR. Tetrahedron. Asymmetry 5, 699 (1994).

27. Pan, W. et al. $\mathrm{Cs}_{2} \mathrm{AgBiBr}_{6}$ single-crystal $\mathrm{X}$-ray detectors with a low detection limit. Nat. Photonics 11, 726 (2017).

28. Dolomanov, O. V. et al. OLEX2: a complete structure solution, refinement and analysis program. J. Appl. Crystallogr. 42, 339 (2009).

29. Du, K.-z. et al. Two-dimensional lead (II) halide-based hybrid perovskites templated by acene alkylamines: crystal structures, optical properties, and piezoelectricity. Inorg. Chem. 56, 9291 (2017).

30. Saidaminov, M. I., Mohammed, O. F. \& Bakr, O. M. Low-dimensional-networked metal halide perovskites: the next big thing. ACS Energy Lett. 2, 889 (2017).
31. Ben-Moshe, A., Teitelboim, A., Oron, D. \& Markovich, G. Probing the interaction of quantum dots with chiral capping molecules using circular dichroism spectroscopy. Nano Lett. 16, 7467 (2016).

32. Govorov, A. O. et al. Theory of circular dichroism of nanomaterials comprising chiral molecules and nanocrystals: plasmon enhancement, dipole interactions, and dielectric effects. Nano Lett. 10, 1374 (2010).

33. Tanaka, K. et al. Electronic and excitonic structures of inorganic-organic perovskite-type quantum-well crystal $\left(\mathrm{C}_{4} \mathrm{H}_{9} \mathrm{NH}_{3}\right)_{2} \mathrm{PbBr}_{4}$. Jpn. J. Appl. Phys. 44, 5923 (2005)

34. Tohgha, U. et al. Ligand induced circular dichroism and circularly polarized luminescence in CdSe quantum dots. ACS Nano 7, 11094 (2013).

35. Sasaki, T. et al. Construction of Chiral Polar Crystals from Achiral Molecules by Stacking Control of Hydrogen-Bonded Layers Using Type II Halogen Bonds. Cryst. Growth Des. 16, 1626 (2016).

36. Yeom, J. et al. Chiromagnetic nanoparticles and gels. Science 359, 309 (2018).

37. Yang, Y. et al. Emergent properties of an organic semiconductor driven by its molecular chirality. ACS Nano 11, 8329 (2017).

38. Odenthal, P. et al. Spin-polarized exciton quantum beating in hybrid organicinorganic perovskites. Nat. Phys. 13, 894 (2017)

39. Niesner, D. et al. Giant rashba splitting in $\mathrm{CH}_{3} \mathrm{NH}_{3} \mathrm{PbBr}_{3}$ organic-inorganic perovskite. Phys. Rev. Lett. 117, 126401 (2016).

40. Hooge, F., Kleinpenning, T. \& Vandamme, L. Experimental studies on 1/f noise. Rep. Prog. Phys. 44, 479 (1981).

41. Gao, L. et al. Passivated single-crystalline $\mathrm{CH}_{3} \mathrm{NH}_{3} \mathrm{PbI}_{3}$ nanowire photodetector with high detectivity and polarization sensitivity. Nano Lett. 16, 7446 (2016).

42. Wang, H. \& Kim, D. H. Perovskite-based photodetectors: materials and devices. Chem. Soc. Rev. 46, 5204 (2017).

\section{Acknowledgements}

This work was financially supported by the National Key R\&D Program of China (2016YFB0700702, 2016YFA0204000), the National Natural Science Foundation of China (61725401 and 51761145048), and the HUST Key Innovation Team for Interdisciplinary Promotion (2016JCTD111 and 2017KFXKJC003) and by the Fundamental Research Funds for the Central Universities (2017KFXKJC0020). We would thank the Analytical and Testing Center of HUST and the Center for Nanoscale Characterization and Devices of WNLO.

\section{Author contributions}

J.T. supervised the whole project. C.C., L.G. and J.T. conceived the idea, designed the experiments, and analyzed the data. C.C., W.G. and C.G. carried out the device fabrication and characterizations. X.D. and Y.Y. assisted in the measurement of photodetector performance and single-crystal diffraction, respectively. Z.L. G.N. and G.L. participated in discussions and gave much helpful suggestion. C.C. and J.T. wrote the paper; all authors commented on the manuscript.

\section{Additional information}

Supplementary Information accompanies this paper at https://doi.org/10.1038/s41467019-09942-z.

Competing interests: The authors declare no competing interests.

Reprints and permission information is available online at http://npg.nature.com/ reprintsandpermissions/

Journal peer review information: Nature Communications thanks the anonymous reviewers for their contribution to the peer review of this work.

Publisher's note: Springer Nature remains neutral with regard to jurisdictional claims in published maps and institutional affiliations.

cc) (i) Open Access This article is licensed under a Creative Commons Attribution 4.0 International License, which permits use, sharing, adaptation, distribution and reproduction in any medium or format, as long as you give appropriate credit to the original author(s) and the source, provide a link to the Creative Commons license, and indicate if changes were made. The images or other third party material in this article are included in the article's Creative Commons license, unless indicated otherwise in a credit line to the material. If material is not included in the article's Creative Commons license and your intended use is not permitted by statutory regulation or exceeds the permitted use, you will need to obtain permission directly from the copyright holder. To view a copy of this license, visit http://creativecommons.org/ licenses/by/4.0/

(C) The Author(s) 2019 